\title{
Study on Unconventional Superconductors via Angle-resolved Specific Heat
}

\author{
Tuson Park \\ Los Alamos National Laboratory, Los Alamos, New Mexico 87545 \\ M. B. Salamon \\ Department of Physics and Materials Research Laboratory, \\ University of Illinois at Urbana-Champaign, Urbana, Illinois 61801
}

(Dated: October 24, 2018)

\begin{abstract}
The gap function in unconventional superconductors may vanish at points or lines in momentum space, permitting electronic excitations, termed nodal quasiparticles, to exist at temperatures well below the superconducting transition. In the vortex phase, the presence of nodal quasiparticles should be directly observable through the variation of the heat capacity with the angle between a magnetic field and the location of the zeroes of the gap. The heat capacity of candidate non-magnetic unconventional superconductors $\mathrm{Lu}(\mathrm{Y}) \mathrm{Ni}_{2} \mathrm{~B}_{2} \mathrm{C}$ were found to exhibit fourfold oscillations with field angle, the first such observation. The observed angular variations are in quantitative agreement with theory, confirming that quasiparticles are created via Doppler shifts at nodes along $<100>$. Anomalous disorder effects have been also observed in the field-angle dependent heat capacity $C_{p}(\alpha)$. In a slightly disordered sample, anomalous secondary minima along $<110>$ appeared for $\mu_{0} H>$ $1 \mathrm{~T}$, leading to an eightfold pattern. The coexistence of an anisotropic superconducting gap and nonlocal effects is shown to drive the anomalous behavior. These results demonstrate that fieldangle-dependent heat capacity can be a powerful tool in probing the momentum-space gap structure in unconventional superconductors such as high $\mathrm{T}_{c}$ cuprates, heavy fermions, borocarbides, etc.
\end{abstract}

\section{INTRODUCTION}

Most superconductors behave conventionally; electronic excitations are suppressed by the BCS gap causing the electronic heat capacity, for example, to be exponentially small at temperatures well below the superconducting transition. Recently, a number of superconductors, cuprates $\frac{1}{2}$ and heavy-fermion metals ${ }^{2}$ among them, have been found to be unconventional in that they exhibit gapzero (or nodal) points or lines in momentum space. Electronic excitations - nodal quasiparticles (nqp) - are then observed at low temperatures, giving rise to power-law rather than exponential, behavior. Unlike gapless superconductivity, which can occur in conventional superconductors, the Fermi momenta of these quasiparticles are restricted to nodal regions of the Fermi surface, giving a strong directional dependence to various physical properties.

In 1992, Yip and Sauls proposed a nonlinear Meissner effect in the penetration depth of cuprate high- $T_{c}$ superconductors ${ }^{3}$ Since the effect uses the field-direction dependence of the supercurrent in locating the positions of the nodal lines (or points) of an unconventional gap in momentum space, it is a stronger test of the symmetry of the superconducting state than power-law behavior for the penetration depth. The search for the fundamental manifestation of d-wave symmetry has been very controversial from an experimental point of view ${ }^{4.5 .6 .7 .8} \mathrm{Li}$ et $a l$. questioned the observability of the nonlinear Meissner effect $\stackrel{9}{\underline{9}}$ They introduced nonlocal electrodynamics for a d-wave superconductor and found that the local nonlinear Meissner effect is not observable for fields below a crossover scale $H^{*}$ describing the competition between nonlinear and nonlocal effects in the Meissner state. For most orientations of the screening current, the crossover is of the same order as or greater than the lower critical field $H_{c 1}$.

In conventional type II superconductors, the vortex cores play a crucial role in transport properties because a relatively high density of bound states is created there by the suppression of the local order parameter and extended quasiparticle states are completely gapped. In a new symmetry class such as cuprate superconductors, however, Hirschfeld et al. argued that extended quasiparticle states are dominant over vortex bound states because of the presence of the nodes $\frac{10}{10}$ The anisotropy in the gap $\Delta_{k}$ in momentum space fixed to the crystal axes will induce an angular anisotropy in the current response due to the coupling between quasiparticles and supercurrent, leading to the fourfold angular variation in field-angle thermal conductivity of unconventional superconductors with d-wave order parameter. In 1995, Yu and Salamon et al. demonstrated for the first time that the heat transport of YBCO oscillates with in-plane field angle $\stackrel{11,12}{r}$ Subsequent experiments have confirmed the feature and thermal conductivity has been established as a tool that can probe the momentum-specific gap nature in unconventional superconductors $\stackrel{13.14 .15}{=}$ Such measurements, however, are complicated by the competition between Andreev-scattering and Doppler-shift effects.

A much more direct, and far more difficult, experiment is to detect the low-energy density of states of the nodal quasiparticles through its modulation by an in-plane field. Heat capacity provides such a measure. Working in the 2D limit, Vekhter et al. have shown that the density of states of a d-wave superconductor exhibits four-fold oscillation with field angle with respect to crystal axes $\stackrel{16}{16}$ From the experimental stand point, the search 
for the oscillation has been puzzling ${ }_{17,18}^{18}$ The expected $30 \%$ of oscillation amplitude is within the reach of experimental error, but the effect has proven elusive. Whelan and Carbotte argued that the Zeeman energy splitting $\mu H$ competes against the anisotropy $\frac{19}{19}$ At a critical field $H_{c}$, Zeeman and Doppler energy scales become comparable and the DOS variation with field angle vanishes. Recently, Won and Maki used a semi-classical approximation and extended the 2D Vekhter model to a 3D superconductor with modulated cylindrical Fermi surface by including quasiparticles with an out-of-plane momentum component ${ }^{20}$ The $3 \mathrm{D}$ effect causes a strong suppression of the oscillation amplitude to around $6 \%$, making the observation much more difficult.

In this paper, we review the direct observation of a phenomenon that should be common to many so-called unconventional superconductors, those whose Cooper pairs have symmetries more complex than originally envisaged by Bardeen, Cooper and Shrieffer in their classic theory of superconductivity. If, in particular, the pairs have the symmetry similar to d-orbitals in atoms, there will be certain directions in momentum space in which electronic excitations, termed quasiparticles, can arise without an energy gap.

In section 2, the experimental method, sample preparation and characterization will be briefly explained. In section 3, the field-angle heat capacity of $\mathrm{LuNi}_{2} \mathrm{~B}_{2} \mathrm{C}$ is shown to be similar to results reported previously for $\mathrm{YNi}_{2} \mathrm{~B}_{2} \mathrm{C} 30$ The magnetic field dependence will be analyzed by a Won-Maki extension of the Vekhter et al. calculation. The heat capacity dependence on magnetic field angle will be discussed in terms of 3D nodal quasiparticle theory and used as a piece of confirming evidence that $\mathrm{LuNi}_{2} \mathrm{~B}_{2} \mathrm{C}$ and $\mathrm{YNi}_{2} \mathrm{~B}_{2} \mathrm{C}$ belong to a class of superconductors with nodes in gap function. An eightfold pattern appeared in a slightly disordered Lu1221 supports the coexistence of nonlocal effects and anisotropic gap effects. Then, concluding remarks will follow in section 4.

\section{EXPERIMENTS}

The electronic specific heat is a fundamental physical quantity that measures the electronic density of states (DOS) directly and is a bulk property. As noted in the Introduction, it was recently suggested that the specific heat can be used to study the angle-resolved gap structure of unconventional superconductors by studying the DOS variation with magnetic field angle ${ }^{16}$ Considerable efforts have been expended to detect the field-angle variation arising from the anisotropic gap structure in unconventional superconductors, especially in $\mathrm{YBCO}$ Those efforts, however, have suffered from intrinsic experimental limitations. First, a very limited set of field directions were investigated. Typically, the crystal-axes and diagonal directions were chosen because they give the largest contrast in DOS for pure $d_{x^{2}-y^{2}}$ or $d_{x y}$ pairing

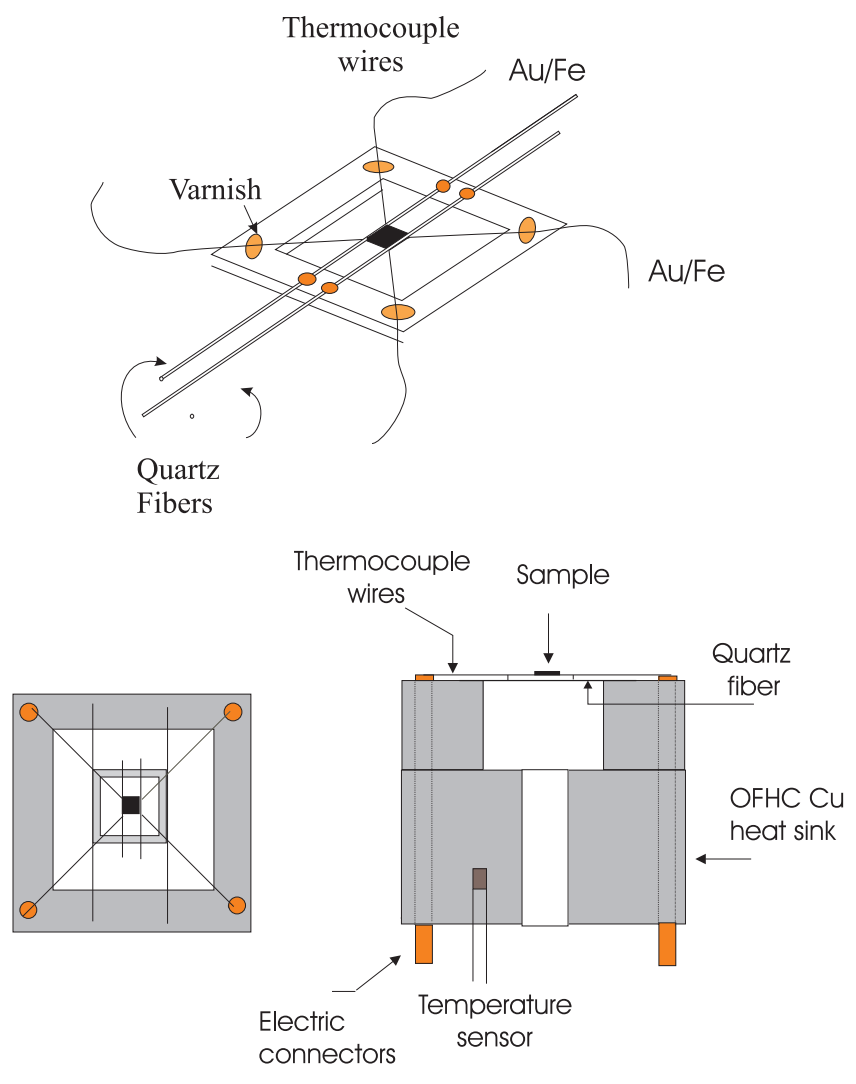

FIG. 1: Sample assembly in ac calorimetry

symmetry. If the pairing symmetry is the mixture of two order parameters, such as $d+s$-wave, then the two directions do not represent gap-maximum or gap-zero (nodes) directions because the nodes move away from the diagonal directions. Second, temperature-variable heat capacity intrinsically contains large background contributions such as lattice vibrations or thermally excited quasiparticles. The large backgrounds cause an uncertainty in extracting the field-induced part, and can easily obscure the small angular variation.

With those problems in mind, we built a new specific heat probe designed exclusively to study the gap structure of superconductors in momentum space. Instead of varying temperature as was done in many previous works, we fixed temperature and magnetic field intensity and made the field angle as an only variable. This experimental scheme enabled us to study the small angular oscillation, which is typically less than $1 \%$ of the measured heat capacity. Some of the key ingredients to this scheme are as following. First, temperature and magnetic field should be very stable. Otherwise, even a small fluctuation could obscure the small change with angle. A Physical Properties Measurement System (PPMS) by Quantum Design was used as a cryogenics platform to satisfy those conditions. Second, a stepping motor with a gear ratio 1:141 was used to rotate the sample and was controlled by a driver from PPMS. Instead of measuring limited directions, we were able to study the angle de- 


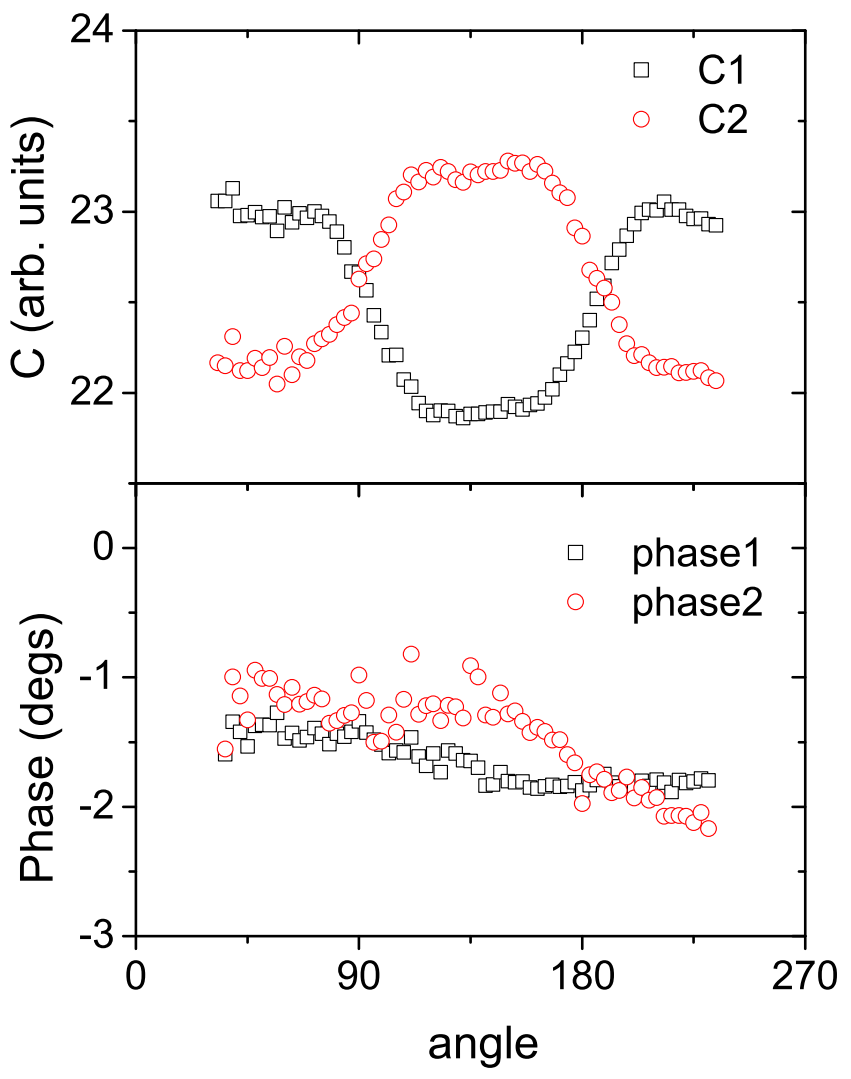

FIG. 2: Upper panel shows the field-angle heat capacity of optimally doped YBCO at $1.7 \mathrm{~K}$ and $7 \mathrm{~T}$. The phase shift with field angle is shown in the lower panel. In both panels, the squares describe the data measured by the first pair of thermocouple wires and the circles by the second pair.

pendence of heat capacity in increments of $3^{\circ}$ or smaller. Third, the heat capacity measurement should be sensitive enough to pick up the minute change with field angle. In addition, the measurement must be able to handle small single crystals because their weight is usually less than $1 \mathrm{mg}$. AC calorimetry was chosen because it has a sensitivity of up to $0.01 \%$ of the measured heat capacity as well as a capability to measure even microgram $(\mu g)$ samples ${ }^{21}$

The $a c$ method used here is an adaptation of the Sullivan and Seidel technique ${ }^{22}$ Fig. 1 shows the sample assembly. The front face of the sample was coated with colloidal graphite suspension (DAG) thinned with isopropyl alcohol to prevent a possible change of the optical absorptions of the sample. The sample was weakly coupled to the heat bath through helium gas and suspending thermocouple wires. At high temperatures, the thermal coupling can be adjusted mainly by controlling the amount of the helium gas surrounding the sample, while the thermal leak through thermocouple wires should be taken into account at cryogenic temperatures. As a heating source, infrared laser light $(\lambda=789.2 \mathrm{~nm})$ was used. The light was modulated electronically to make a square-wave pulse and was guided into the PPMS sample space through

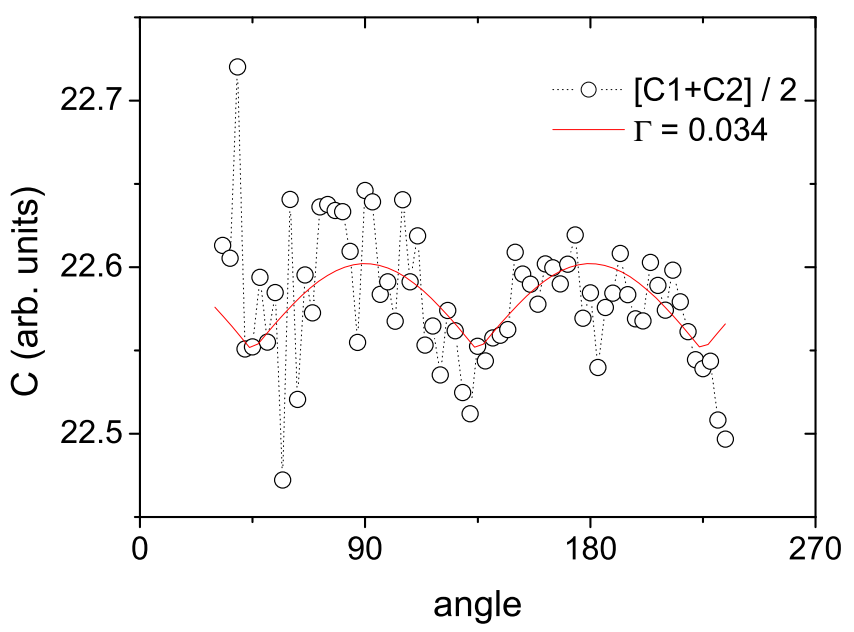

FIG. 3: The averaged field-angle heat capacity of YBCO at 1.7 $\mathrm{K}$ and $7 \mathrm{~T}$. The solid line shows a theoretical calculation of fourfold oscillation with the oscillation amplitude of $3.4 \%$ (see text).

an optical fiber. The oscillating heat input incurred a steady temperature offset (or $d c$ offset) against the heat bath with an oscillating temperature superposed. The measured oscillation temperature $T_{a c}$ was converted to heat capacity by the relationship $C \propto 1 / T_{a c}$ in a proper frequency range.

In our field-angle heat capacity measurements, two thermocouple pairs were used to measure the temperature oscillations $T_{a c}$ simultaneously. The two sets of $\mathrm{Au} / \mathrm{Fe}$ and chromel thermocouple wires were configured so that they were $90^{\circ}$ apart, which enabled us to rule out the field-angle contribution from the thermocouple wires due to field-dependent thermopower of $\mathrm{Au} / \mathrm{Fe}$. If the angular oscillations come from our experimental setup, the two signals will be out of phase while they will be in phase with each other if they come from sample. As an example, we studied an optimally doped YBCO (Fig. 2). The squares in the top panel describe the angular heat capacity measured by the first pair of the thermocouple wires and the circles by the second pair. The two sets of data were measured simultaneously at $1.7 \mathrm{~K}$ in $7 \mathrm{~T}$ with magnetic field rotating within the $\mathrm{CuO}_{2}$ plane of YBCO. Both of them show clear two-fold oscillations with field angle measured against a-axis. Note that the oscillations are out of phase with each other, indicating that they come from the thermocouple wires. The bottom panel of Fig. 2 shows the phase shift with field angle. There is no correlation with the heat capacity shown in the upper panel, confirming that the two-fold oscillation of the heat capacity is not related to intrinsic sample properties ${ }^{23}$

In Fig. 3, we show the averaged heat capacity of YBCO $\left(2 C_{\text {ave }}=C_{1}+C_{2}\right)$. As expected, the two-fold components are almost suppressed. Now that the artifact from the experimental setup is removed, we can study the intrinsic sample property. The solid line describes a fit by a cusped fourfold variation with oscillation amplitude of 
$3.4 \%$, i.e., $C(\alpha)=C_{0}+c_{4}(1+\Gamma|\sin 2 \alpha|)$. Here, $\alpha$ is the magnetic field angle with respect to the $a$-axis and $\Gamma$ is the oscillation amplitude. The periodicity is $\pi / 4$ and the minima are located at $\langle 110\rangle$ directions, which are consistent with $d_{x^{2}-y^{2}}$-wave order parameter in YBCO where the nodes are located along $\langle 110\rangle$. The field-angle oscillation, however, is obscured by noise which is comparable to the angular change.

\section{OBSERVATION OF NODAL QUASIPARTICLES AND NONLOCAL EFFECTS IN THE NONMAGNETIC SUPERCONDUCTORS $\mathbf{L U}(\mathbf{Y}) \mathrm{NI}_{2} \mathrm{~B}_{2} \mathrm{C}$}

The discovery of $\mathrm{RNi}_{2} \mathrm{~B}_{2} \mathrm{C}(\mathrm{R}=\mathrm{Y}, \mathrm{Lu}, \mathrm{Tm}, \mathrm{Er}$, Ho, and Dy) captured attention of the superconductivity community because of its relatively high transition temperature $\left(T_{c}\right)$ and the coexistence of magnetism and superconductivity 24.25 .26 The highest $T_{c}$ 's in the borocarbide family are $16.5 \mathrm{~K}$ and $15.5 \mathrm{~K}$ in the nonmagnetic members with $\mathrm{Lu}$ and $\mathrm{Y}$ elements respectively. Amid the controversy over the pairing symmetry of the borocarbides, there is growing evidence that the gap function is highly anisotropic and possibly has nodes where the gap becomes zero in momentum space. In specific heat measurements, a power law behavior was observed in the temperature dependence and the electronic coefficient $\gamma(H)$, to the extent it can be extracted, follows a squareroot field dependence, 27.28 Thermal conductivity measurements showed low energy excitations at as low as 70 $\mathrm{mK}$ and an anomalous magnetic field dependence similar to the unconventional superconductor $\mathrm{UPt}_{3}$ but different from the exponential dependence characteristic of an isotropic s-wave gap ${ }^{29}$ Compelling evidence for the presence of nodes along $\langle 100\rangle$ directions has been reported from both z-axis field-angle thermal conductivity $\underline{15}$ and field-angle heat capacity measurements of $\mathrm{YNi}_{2} \mathrm{~B}_{2} \mathrm{C}, 30$

Recently, a magnetic field-driven flux line lattice (FLL) transition has been observed both in the magnetic member (Er) and in the nonmagnetic members ( $\mathrm{Y}$ and $\mathrm{Lu}$ ) of the tetragonal borocarbides $\frac{31.32 .33 .34}{3 h e}$ transition from square to hexagonal vortex lattice occurs due to the competition between sources of anisotropy and vortexvortex interactions. The repulsive nature of the vortex interaction favors the hexagonal Abrikosov lattice, whose vortex spacing is larger than that of a square lattice. The competing anisotropy, which favors a square lattice, can be due to lattice effects (fourfold Fermi surface anisotropy) 35 or unconventional superconducting order parameter ${ }^{36}$ In this section, we present evidence that both the gap anisotropy and nonlocal effects coexist and the coexistence is the origin of the anomalous crossover from a fourfold pattern to an eightfold pattern in $C(\alpha)$ with increasing field in $\mathrm{LuNi}_{2} \mathrm{~B}_{2} \mathrm{C}$.

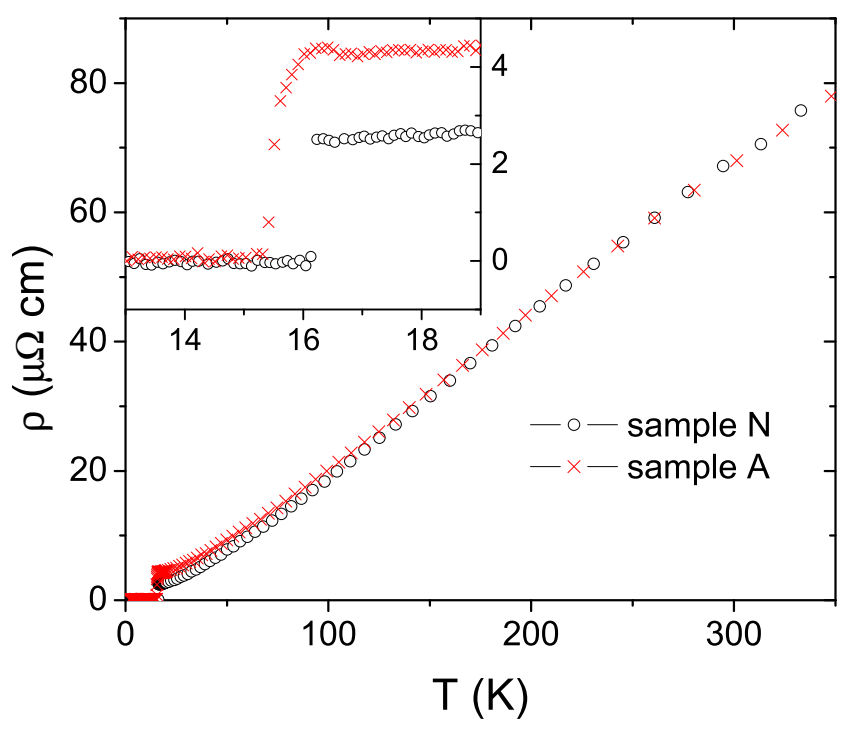

FIG. 4: Temperature dependence of the in-plane resistivity of sample A (crosses) and sample N (circles).

\section{A. Disorder effects}

The best samples of $\mathrm{LuNi}_{2} \mathrm{~B}_{2} \mathrm{C}$ exhibit behavior identical to that reported earlier for $\mathrm{YNi}_{2} \mathrm{~B}_{2} \mathrm{C}, \underline{30}$ but disordered samples are significantly different. Three single crystals of $\mathrm{LuNi}_{2} \mathrm{~B}_{2} \mathrm{C}$ from different batches were studied. Sample N was annealed at $T=1000 \mathrm{C}^{\circ}$ for 100 $\mathrm{h}$ under high vacuum, while sample $\mathrm{A}$ and $\mathrm{C}$ were not annealed. The annealed crystal, sample N, showed the highest $T_{c}(16.1 \mathrm{~K})$ and residual resistivity ratio $(\mathrm{RRR})$ of 27 , while the unannealed sample A recorded the lowest $T_{c}$ of $15.5 \mathrm{~K}$ and RRR of 16 (see Fig. 4), suggesting a judicious postgrowth annealing may improve the sample quality. The resistivity at $T_{c}$ is 2.34 and $1.44 \mu \Omega \cdot \mathrm{cm}$ for samples $\mathrm{A}$ and $\mathrm{N}$, corresponding to mean-free paths of 144.5 and $234 \AA$ respectively. Assuming that $16.1 \mathrm{~K}$ is the $T_{c}$ for a pure sample, sample $\mathrm{A}$ is equivalent to $0.8 \%$ of $\mathrm{Co}$ doping on the $\mathrm{Ni}$ site; i.e., $\mathrm{Lu}\left(\mathrm{Ni}_{1-x} \mathrm{Co}_{x}\right)_{2} \mathrm{~B}_{2} \mathrm{C}$ with $x=0.008 \stackrel{37}{3}$ Sample $\mathrm{C}$ with $T_{c}=15.8 \mathrm{~K}$ is less disordered than sample A.

For an isotropic s-wave superconductor, the contribution to the density of states (DOS) comes from the localized states in the vortex core. Since the density of vortices is proportional to the magnetic field, the fieldinduced DOS is linearly dependent on the field and $\beta=1$. For superconductors with line nodes, delocalized states leaking through the nodes dominate the DOS. When we integrate the contribution in a vortex unit cell and multiply by the density of vortices, the electronic DOS is proportional to $\sqrt{H}$ in an intermediate region, i.e. $H_{c 1} \ll H \ll H_{c 2}$, where $H_{c 2}$ is upper critical field. This so called Volovik effect gives $\beta=0.5$ for line node and $\beta=1$ for point node ${ }^{28}$ Recently, there have been several reports that s-wave superconductors such as $\mathrm{CeRu}_{2}{ }^{39}$ and $\mathrm{NbSe}_{2}{ }^{27}$ behave like d-wave superconductors, show- 
ing sub-linear magnetic field dependence in specific heat. Ichioka et al. 40 extended the Volovik calculation to the entire region, i.e. $H_{c 1}<H<H_{c 2}$. While the Volovik effect considered the delocalized states only, they included the localized states bound in a vortex core and the quasiparticle transfers between vortices. In their numerical calculation, the DOS dependence in the d-wave pairing states gives an exponent $\beta=0.41$, rather than Volovik's $\beta=0.5$. As for those with anisotropic s-wave pairing symmetry, the exponent becomes 0.67 , which is clearly distinguishable from 0.5 or 0.41 .

The disorder effects are also manifested in the magnetic field dependence of the electronic specific heat $C_{e l}$. In pure samples (sample $\mathrm{C}$ and $\mathrm{N}$ ), the low-temperature specific heat shows a $\sqrt{H}$ dependence in the mixed state, but there is a deviation from the $\sqrt{H}$ dependence at a finite field in the disordered sample A. Fig. 5(a) shows the heat capacity of sample $\mathrm{C}$ at $2.5 \mathrm{~K}$ with increasing (circles) and decreasing (crosses) magnetic field along [100] direction. The dashed line which represents the least square fit of $C_{0}+b\left(H-H_{0}\right)^{\beta}$ best describes the field dependence with $\beta=0.46$. Here $C_{0}$ is the zero-field heat capacity and the offset $H_{0}$ is essentially a lower critical field $\left(H_{c 1}\right)$ and $0.1 \mathrm{~T}$ was used for the least-square fit.

Figures 5(b)-(d) show the heat capacity of the disordered sample $\mathrm{A}$ at $2.5,4$, and $8 \mathrm{~K}$ respectively. Low-field data were best described by the square-root field dependence (dashed lines) and the arrows indicate the points where the data deviate from the fit. The deviation field shows a systematic increase with temperature, i.e, $0.8 \mathrm{~T}$ at $2.5 \mathrm{~K}, 1.8 \mathrm{~T}$ at $4 \mathrm{~K}$, and no clear deviation at $8 \mathrm{~K}$. Above the deviation field, the data fall below the $\sqrt{H}$ line.

\section{B. Angle-resolved Specific Heat}

In addition to the $\sqrt{H}$ dependence in unconventional superconductors, the density of states $N(H, \alpha)$ also depends on the orientation of the superfluid flow with respect to the nodes, which results in angular oscillation in $C(\alpha)$ 16.41 The supercurrent flow around a vortex leads to a Doppler energy shift, $\delta E=\mathbf{v}_{s} \cdot \hbar \mathbf{k}_{F}$, where $\mathbf{v}_{s}$ is the velocity of the superfluid and $\hbar \mathbf{k}_{F}$ is the Fermi momentum of nodal quasiparticles. When the field direction is normal to the plane containing nodes, the DOS is the average over the whole Fermi surface, leading to a square root field dependence. When the field is in the nodal plane, however, the Doppler shift has a field-direction dependence as well, $\delta E \approx \frac{E_{h}}{\rho} \sin \beta \sin (\phi-\alpha), 16$ Here $\phi$ is an azimuthal angle of the gap node and $\beta$ is a vortex current winding angle. The energy scale associated with the Doppler effect is defined as

$$
E_{h}=\frac{a \hbar v^{*}}{2}\left(\frac{\pi H}{\Phi_{0}}\right)^{1 / 2}
$$

where the geometrical constant $a$ is order of unity, $v^{*}=$ $\sqrt{v_{a} v_{c}}$ is the average Fermi velocity, and $\Phi_{0}$ is the flux (a) Sample C at $2.5 \mathrm{~K}$

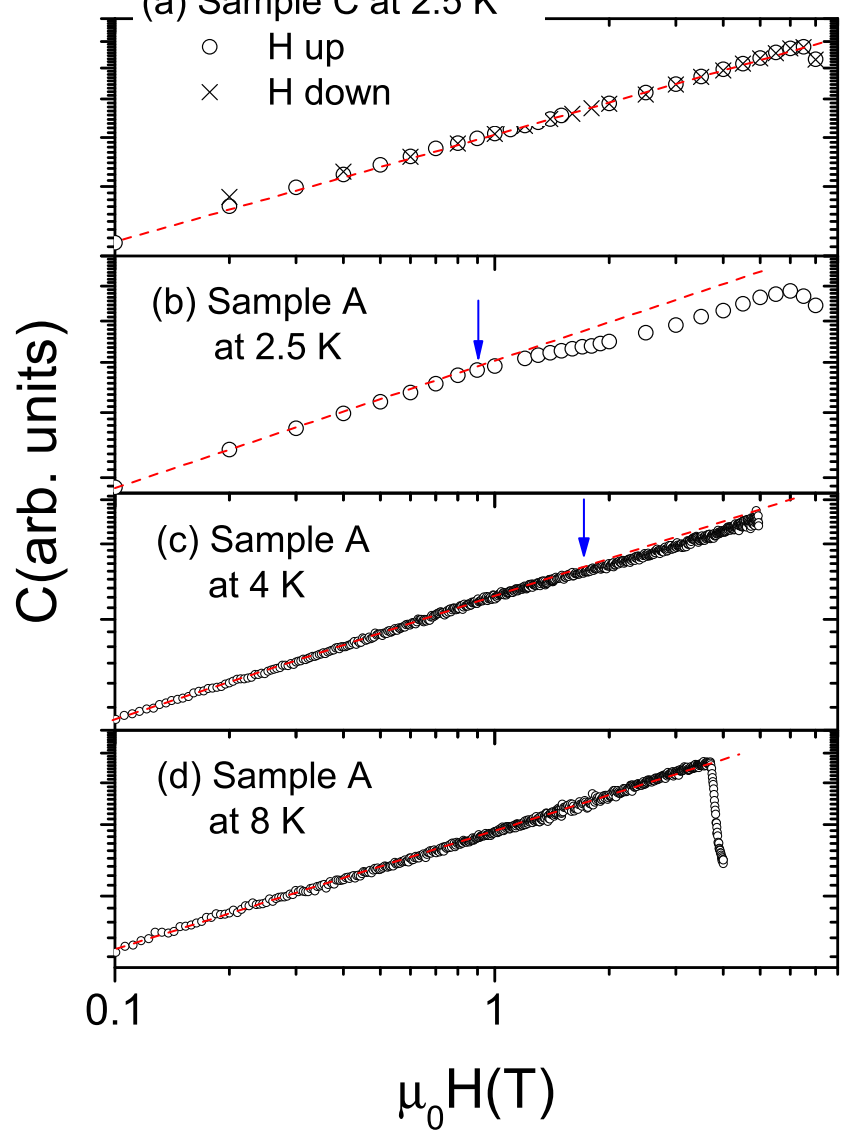

FIG. 5: (a) Heat capacity of sample $C$ at $2.5 \mathrm{~K}$ for $H \|[110]$. (b)-(d) Heat capacity of sample $A$ at $2.5,4$, and $8 \mathrm{~K}$, respectively for $H \|[110]$. Dashed lines are $H^{1 / 2}$ fits and arrows indicate the deviation points from the $H^{1 / 2}$ dependence. ${ }^{38}$

quantum. The variable $\rho=r / R$, where $r$ is the distance from the vortex core and $2 R$ is the intervortex distance. Working in the 2D limit, Vekhter et al. calculated the DOS , $N \approx\left(N_{1}+N_{2}\right) / 2$, for an in-plane magnetic field when $\omega, E_{h} \ll \Delta_{0}$ where $\omega$ is the Matsubara frequency, and for four nodes at angles $\alpha_{n}$ from orthogonal axes in a plane :

$$
\frac{N_{i}(\omega, h, \alpha)}{N_{0}}=\left\{\begin{array}{lc}
\frac{\omega}{\Delta_{0}}\left(1+\frac{1}{2 x^{2}}\right) & \left(x=\omega / E_{i} \geq 1\right) \\
\frac{E_{i}}{\pi \Delta_{0} x}\left[\left(1+2 x^{2}\right) \arcsin x+3 x \sqrt{1-x^{2}}\right. & (x \leq 1),
\end{array}\right.
$$

where $\mathrm{i}=1,2, \quad E_{1}=E_{h}\left|\sin \left(\alpha_{n}-\alpha\right)\right|$ and $E_{2}=$ $E_{h}\left|\cos \left(\alpha_{n}-\alpha\right)\right|$. The energy $E_{1}$ is for nodes close to the field direction, while $E_{2}$ is for nodes far from it. $N_{0}$ is the normal-state density of states.

When the magnetic field is along a nodal direction, $\alpha=\alpha_{n}+m \pi / 2$ where $m$ is an integer; the two nodal positions perpendicular to the field contribute fully, while the two parallel to the field do not contribute at all because the Doppler shift is an inner product between the superfluid velocity $\mathbf{v}_{s}$ and the quasiparticle momentum $\hbar \mathbf{k}_{F}$. When the field is along a maximal gap direction $\left(\alpha=\alpha_{n}+(2 m+1) \pi / 4\right)$, in contrast, all four nodal points 
contribute equally to the DOS with a factor of $\sqrt{2}$ less than the full contribution. When we add all the contributions, the total DOS has minima along nodal directions and maxima along maximal gap directions. At $T=0 \mathrm{~K}$, the oscillation is cusped with a contrast of $1 / \sqrt{2}$ between the minima and the maxima and is independent of the magnetic field. At a finite temperature, however, the sharp contrast is washed out due to thermal effects, leading to more rounded minima and the DOS oscillation now depends on the magnetic field.

The oscillation amplitude depends on the gap geometry and dimensionality of the superconductors. The Vekhter et al. model above assumes the Fermi momentum of nodal quasiparticles (nqp's) to be restricted to the nodal plane. A more realistic calculation, therefore, is to allow nqp Fermi momentum out of the nodal planes, which decreases the amplitude of the angular variation. 20 We account for the $3 \mathrm{D}$ effect by replacing the Dopplerrelated energies $\mathrm{E}_{1}$ and $\mathrm{E}_{2}$ by $E_{1}^{3 D}=E_{h}\left[\sin ^{2}\left(\alpha_{n}-\alpha\right)+\right.$ $\left.\cos ^{2} \theta\right]^{1 / 2}$ and $E_{2}^{3 D}=E_{h}\left[\cos ^{2}\left(\alpha_{n}-\alpha\right)+\cos ^{2} \theta\right]^{1 / 2}$ respectively and integrate the DOS over polar angle $\theta$ :

$N(w, h, \alpha)=\frac{1}{2 \pi} \int_{0}^{2 \pi} \frac{1}{2}\left[N_{1}(w, h, \alpha, \theta)+N_{2}(w, h, \alpha, \theta)\right] d \theta$.

The qualitative features are the same as those in the $2 \mathrm{D}$ case except that the oscillation amplitude decreases from $41 \%$ to $6 \%$ in $3 \mathrm{D}$ superconductors at $T=0 \mathrm{~K}$. The $3 \mathrm{D}$ effect was indeed confirmed experimentally in the field-angle heat capacity measurement of $\mathrm{YNi}_{2} \mathrm{~B}_{2} \mathrm{C}, 30 \mathrm{We}$ note that a similar effect is predicted for an $(\mathrm{s}+\mathrm{g})$-wave superconductor 42

The upper panel of Fig. 6 shows the field-directional angular dependence of the total heat capacity at $2 \mathrm{~K}$ and $0.6 \mathrm{~T}$, where the transverse magnetic field was rotated within the basal plane of $\mathrm{LuNi}_{2} \mathrm{~B}_{2} \mathrm{C}$. The total heat capacity consists of constant, 2-fold, and 4-fold contributions: $C_{\text {total }}(\alpha, H)=C_{0}+C_{2}(\alpha, H)+C_{4}(\alpha, H)$. The field-independent constant $C_{0}$ is due to nonmagnetic contributions, such as the lattice heat capacity and thermally excited nqp's, and is determined experimentally from $C(T)$ at $0 \mathrm{~T}$. The 2 -fold contribution $C_{2}(\alpha, H)$ comes from our experimental setup and has a functional form of $c_{2} \cos 2 \alpha$. The $\mathrm{Au} / \mathrm{Fe}$ and chromel thermocouple wire is a major source of this contribution, but misalignment of the basal plane of the sample against the field direction could also lead to the 2 -fold component because of the anisotropy between in-field ab-plane and c-axis heat capacities. The dashed line in the upper panel of the Fig. 6 is $C_{2}(\alpha, H)$; the 2 -fold signal is about $40 \%$ of the 4-fold component at $0.6 \mathrm{~T}$ and increases with magnetic field.

The lower panel of the Fig. 6 shows the $0.6 \mathrm{~T}$ data after subtraction of the 2 -fold and the lattice components : $C_{4}(\alpha, H)=C_{\text {total }}(\alpha, H)-C_{2}(\alpha, H)-C_{0}$. The 4 -fold variation is clearly seen. To make a quantitative analysis, we fit our data to $C_{4}(\alpha, H)=c_{4}(H)(1+\Gamma|\sin 2 \alpha|)$. The coefficient $c_{4}(H)$ and the angular contrast $\Gamma$ were treated

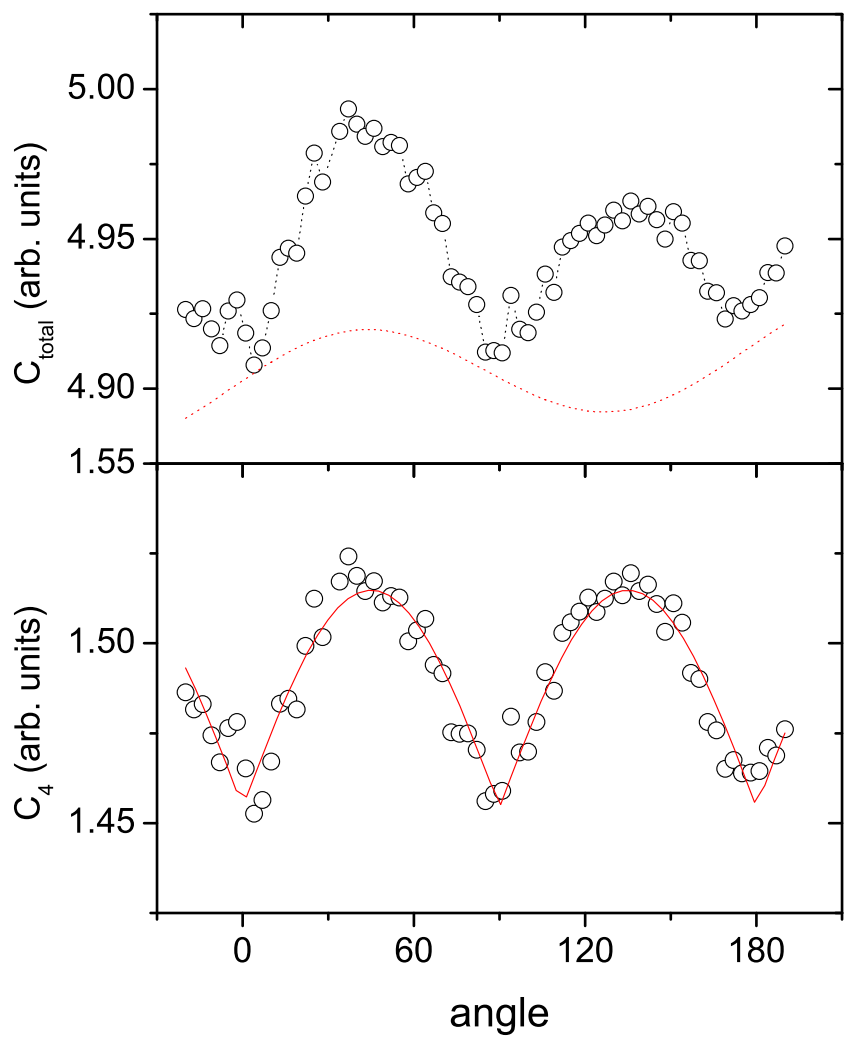

FIG. 6: Field directional dependence of the heat capacity of sample $\mathrm{A}$ at $2 \mathrm{~K}$ and $0.6 \mathrm{~T}$. The field angle $\alpha$ is measured with respect to the a-axis. The top panel shows the total heat capacity (open circles) and the 2-fold component, $C_{2}(\alpha)$ relative to baseline of 4.9 (dashed line). The bottom panel shows the same data after subtraction of the 2-fold and lattice contributions, $C_{4}=C_{\text {total }}-C_{2}(\alpha)-C_{0}$. The data were best described by a cusped function, $C_{4}(\alpha, H)=c_{4}(H)(1+$ $\Gamma|\sin 2 \alpha|)$ with $c_{4}=1.45$ and $\Gamma=0.0415$.

as field dependent fitting parameters. The solid line in the lower panel of Fig. 6 shows the fit with $\Gamma=0.0415$ at $0.6 \mathrm{~T}$. The sharp minima along $<100>$ indicate that there exist gap minima or nodal structures along those directions. The nodal positions and the small oscillation amplitudes are consistent with previous reports of $\mathrm{YNi}_{2} \mathrm{~B}_{2} \mathrm{C},{ }^{15,30}$ The field-angle heat capacities of sample $\mathrm{C}$ and $\mathrm{N}$ at low fields are essentially the same as that of sample A.

Figure 7 gives 3D surface plots of the field-angle heat capacity of sample $\mathrm{C}$ at $2.5 \mathrm{~K}$. The $\mathrm{x}$-axis is the in-plane field direction $\alpha$ with respect to the crystal axes and the $\mathrm{y}$-axis is the applied magnetic field intensity in tesla. The $\mathrm{Z}$-axis is the four-fold heat capacity normalized by the Doppler-induced heat capacity: $C_{4}(H, \alpha) / c_{4}(H)$. Figure $7(\mathrm{a})$ is a numerical calculation of the $3 \mathrm{D}$ nodal quasiparticle theory ${ }^{16,20}$ and Fig. 7(b) are the experimental results of sample C. In the quasiparticle theory, the only adjustable parameter is the Doppler-related energy $E_{h}\left(v^{*}\right)$ (see Eq. 1). In the numerical calculation, we used $a=1$, the gap maximum $\Delta=1.76 k_{B} T_{c}$, and the average Fermi 

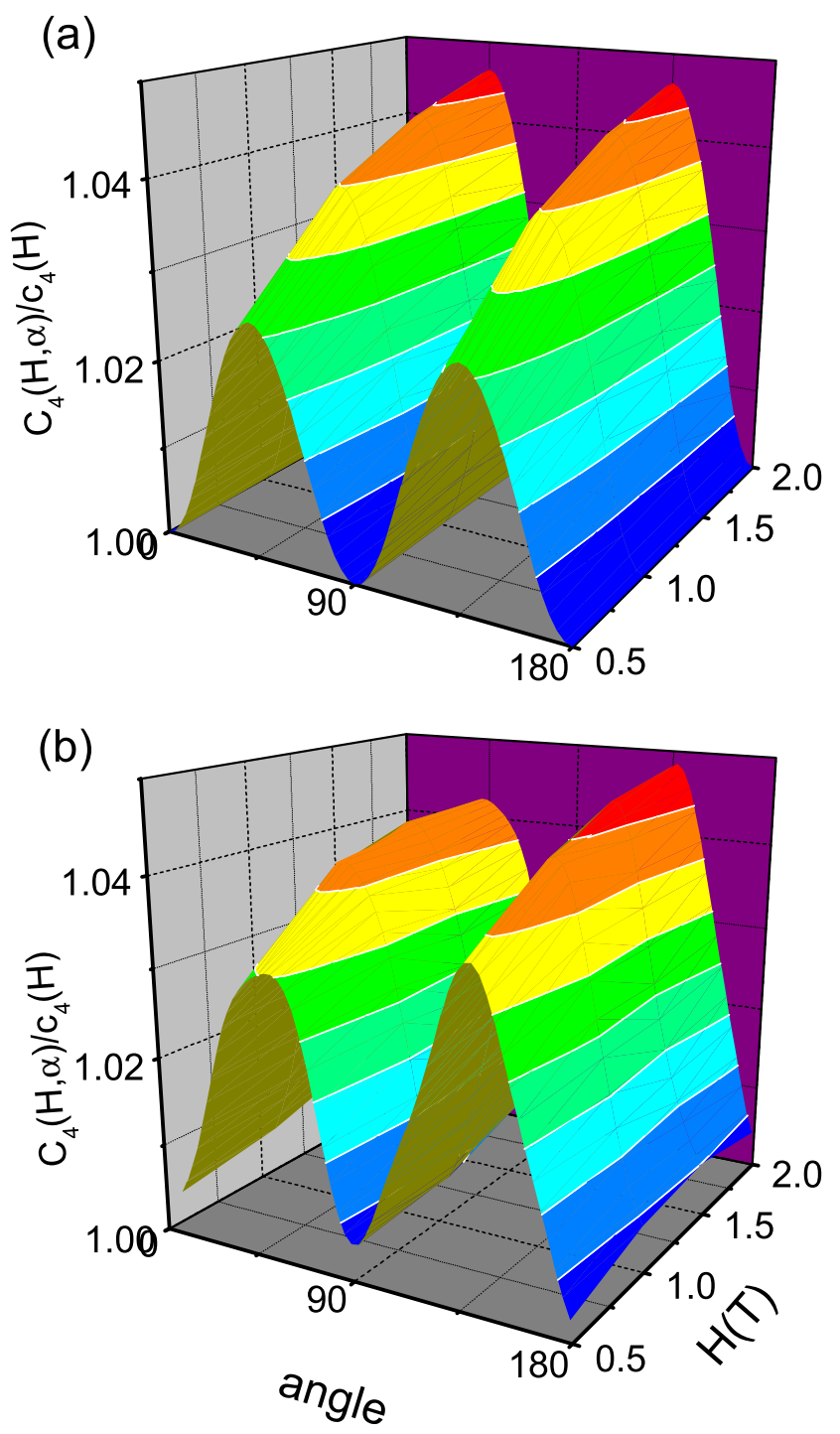

FIG. 7: The 3D surface plots describe magnetic field-angle (xaxis) and field-intensity (y-axis) dependence of the in-plane specific heat ratio at $2.5 \mathrm{~K}: C_{4}(H, \alpha) / c_{4}(H)$. Fig. (a) is a numerical calculation of the specific heat due to nodal quasiparticles in 3D system and Fig. (b) is the experimental data of the single crystal sample C. The experimental data were FFT low-pass filtered with a cut-off frequency of $0.03 \mathrm{~Hz}$ with angles being considered as time. Both plots (a) and (b) were constructed with $0.5,1,1.5$, and $2 \mathrm{~T}$ data sets.

velocity $v^{*}=\sqrt{v_{a} v_{c}}=1.3 \times 10^{7} \mathrm{~cm} / \mathrm{sec}$. The extracted Fermi velocity is similar to that from the band structure calculation $\left(\sqrt{v_{a} v_{c}} \sim 1.2 \times 10^{7} \mathrm{~cm} / \mathrm{sec}\right)$, ${ }^{35}$ The $3 \mathrm{D}$ plots of sample $\mathrm{N}$ show similar behavior to that of sample $\mathrm{C}$ requiring a larger Fermi velocity, i.e., $v^{*}=1.5 \times 10^{7}$ $\mathrm{cm} / \mathrm{sec}^{23}$ The larger value may be due to the uncertainty in determining the field-induced heat capacity.

In field-angle heat capacity measurements, there are three relevant energy scales, which are the Doppler energy scale $E_{h}$, the paramagnetic energy $\mu H$, and the thermal energy of $k_{B} T$. In the mixed state, the paramagnetic

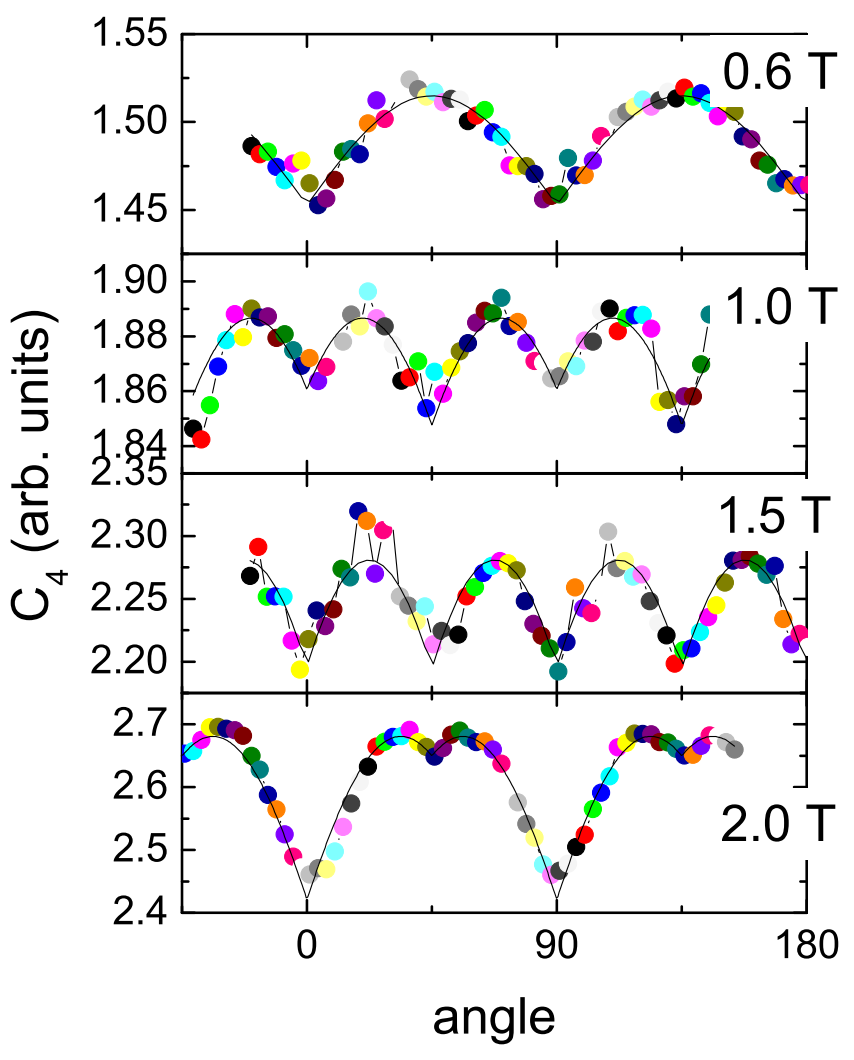

FIG. 8: Angle-resolved heat capacity of sample A at $2 \mathrm{~K}$ and a $4+4$ pattern (solid line). The magnetic fields are 0.6, 1 , 1.5 , and $2 \mathrm{~T}$, from top to bottom panel.

energy is of order $1 \mathrm{~K}$ and the Doppler energy scale is $\sim 10 \mathrm{~K}$. The latter is much higher than the experimental temperature $2 \mathrm{~K}$. Therefore, the Doppler effect on the nodal quasiparticles is dominant over the thermal and the paramagnetic effects in this regime, indicating that the 3D nodal quasiparticle theory of the Doppler effect is applicable as was evident from the reasonable agreement between experiments and the numerical calculations.

Figure 8 shows the heat capacity of sample $\mathrm{A}$ as a function of field angle at $2 \mathrm{~K}$ and $0.6,1.0,1.5$, and $2.0 \mathrm{~T}$ from top to bottom panel. At $1 \mathrm{~T}$, surprisingly, additional minima develop along $<110>$, producing two sets of fourfold patterns or 8-fold, an effect not observed in either sample $\mathrm{C}$ or $\mathrm{N}$ with higher $T_{c}$ 's. The crossover field from the fourfold to the $(4+4)$ pattern of sample A lies between 0.6 and $1 \mathrm{~T}$, which is also the point where the heat capacity of sample A deviates from the $\sqrt{H}$ dependence (see Fig. 5). With increasing field, the splitting gradually disappears and the field-angle heat capacity recovers its fourfold pattern above $4 \mathrm{~T}$. We also measured the field-angle heat capacity of sample $\mathrm{A}$ at $4 \mathrm{~K}$ to check if the anomalous peak splitting persists at higher temperatures (Fig. 9). The fourfold pattern now persists to $1 \mathrm{~T}$, evolving into two sets of fourfold patterns above $2 \mathrm{~T}$. The $4 \mathrm{~T}$ data at $4 \mathrm{~K}$ have a shape similar to the $2 \mathrm{~T}$ data at $2 \mathrm{~K}$. The crossover field $H_{s 1}$ increases with increasing 


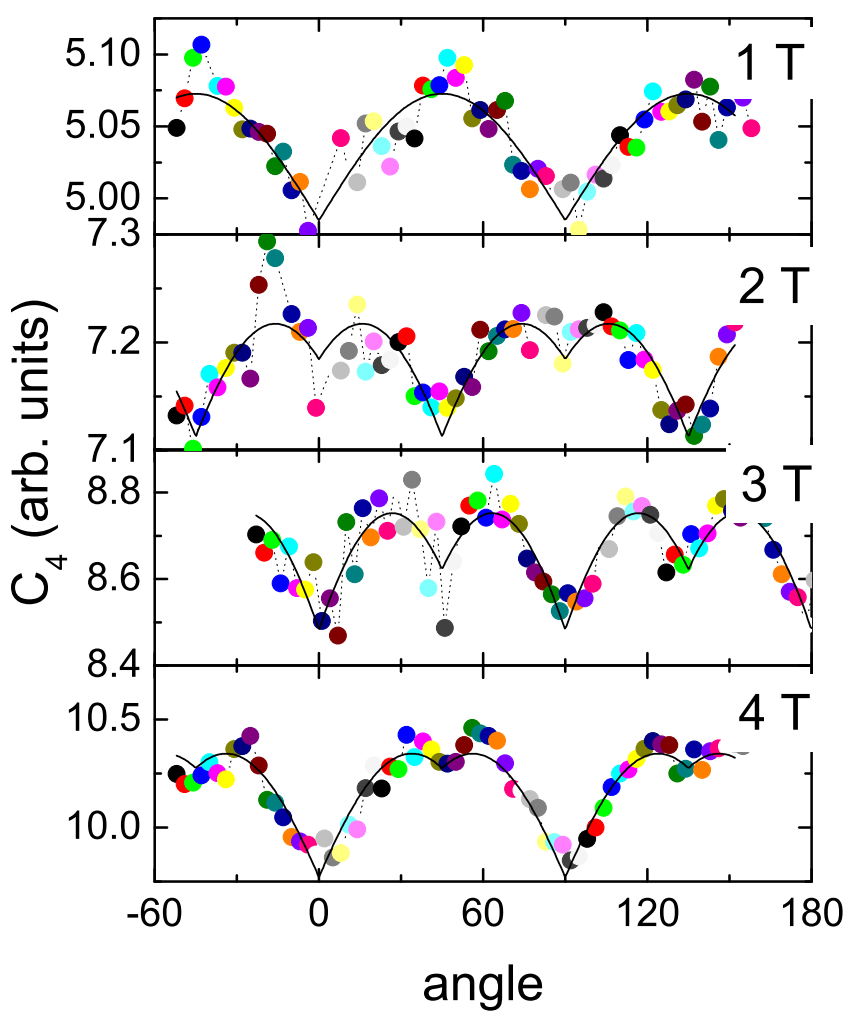

FIG. 9: Angle-resolved heat capacity of sample A at $4 \mathrm{~K}$ and a $4+4$ pattern (solid line). The applied magnetic fields are $1,2,3$, and $4 \mathrm{~T}$, from top to bottom panel.

temperature.

It is worth noting that the anomalous 8-fold pattern occurs only at sample A, which has half the electronic mean free path of the sample $\mathrm{N}$, while the $T_{c}$ is slightly decreased. According to the nonlocal theory by Kogan et al. 35 the hexagonal-to-square FLL transition depends on the electronic mean free path $l$ and the superconducting coherence length $\xi$. Gammel et al. found that a mere $9 \%$ of Co doping onto the $\mathrm{Ni}$ site in Lu1221 can make the FLL transition field 20 times higher than that of the pure matrix for $H \|[001] \stackrel{43}{\underline{n}}$ The FLL transition field for pure Lu1221 is around $0.2 \mathrm{~T}, \underline{44}$ which is well below our measurement range, suggesting the nonlocal effects would not influence the field-angle heat capacity of purer samples. In contrast, the disorder in sample A increases the transition field to at least twice higher than that of sample $\mathrm{N}, \stackrel{43}{\longrightarrow}$ i.e., to a field relevant in the field-angle heat capacity measurement of sample A.

When the magnetic field is rotated within the $a b$-plane, the transition field may differ with different field directions because of the different nonlocal range, i.e., $\xi / l$. The two different transition fields can be characterized by $H_{s 1}$ for $<110>$ and $H_{s 2}$ for $<100>$. As a magnetic field rotates within the $a b$-plane for $H_{s 1} \leq H \leq H_{s 2}$ , the FLL will experience a structural change (or distortion), i.e. hexagonal for $H \|[100]$ and square for $H \|[110]$. Since the borocarbides have nodes on the

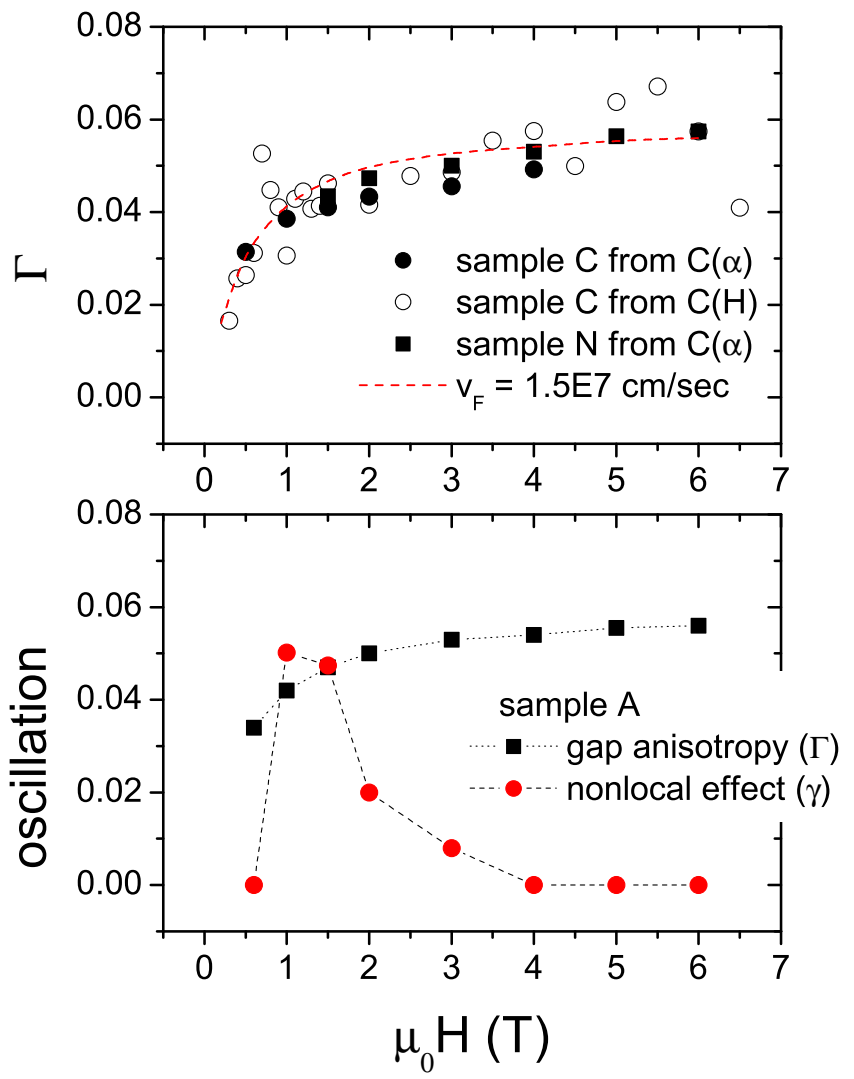

FIG. 10: Oscillation amplitude $\Gamma$ of sample $\mathrm{C}$ (circles) and sample $\mathrm{N}$ (squares). The dashed line is from the $3 \mathrm{D}$ nodal quasiparticle theory with $v_{F}=1.5 \times 10^{7} \mathrm{~cm} / \mathrm{sec}$. The bottom panel contrasts the oscillation amplitude due to the nonlocal effects (circles) and gap anisotropy (squares). ${ }^{38}$

Fermi surface, the DOS will differ depending on the FLL structure ${ }^{40}$ The negative deviation from the $\sqrt{H}$ above $H_{s 1}$ in the heat capacity of sample A (see Fig. 5b) also indicates that the DOS of the hexagonal FLL is larger than that of the square FLL. In addition to the anisotropic gap modulation, 30 the DOS will oscillate due to the FLL anisotropy, leading to a $(4+4)$-fold pattern.

When $H<H_{s 1}$ or $H>H_{s 2}$, the field-induced parameter $c_{4}$ can be considered as constant and the DOS oscillation is just from the gap anisotropy. When $H_{s 1}<$ $H<H_{s 2}$, in contrast, $c_{4}$ is not independent of field direction any more, but oscillates due to the anisotropic FLL transition field: $c_{4}=p 1(1+\gamma|\sin 2(\alpha-45)|) . \quad c_{4}$ has maxima along $<100>$ and minima along $<110>$. Then, the field-angle heat capacity can be written as

$$
C_{4}(\alpha)=p 1(1+\gamma|\sin 2(\alpha-45)|)(1+\Gamma|\sin 2 \alpha|),
$$

where $p 1$ is a field-dependent fitting parameter. The value $\Gamma$ represents the oscillation due to gap anisotropy in pure samples (see Fig. 10). The nonlocal effects give rise to a $45^{\circ}$-shifted 4 -fold pattern and are accounted for by $\gamma$. The solid lines in Fig. 8 and 9 are least square fits of Eq. (4) and represent the data very well. The oscillations due to the nonlocal effects $(\gamma)$ and the gap 


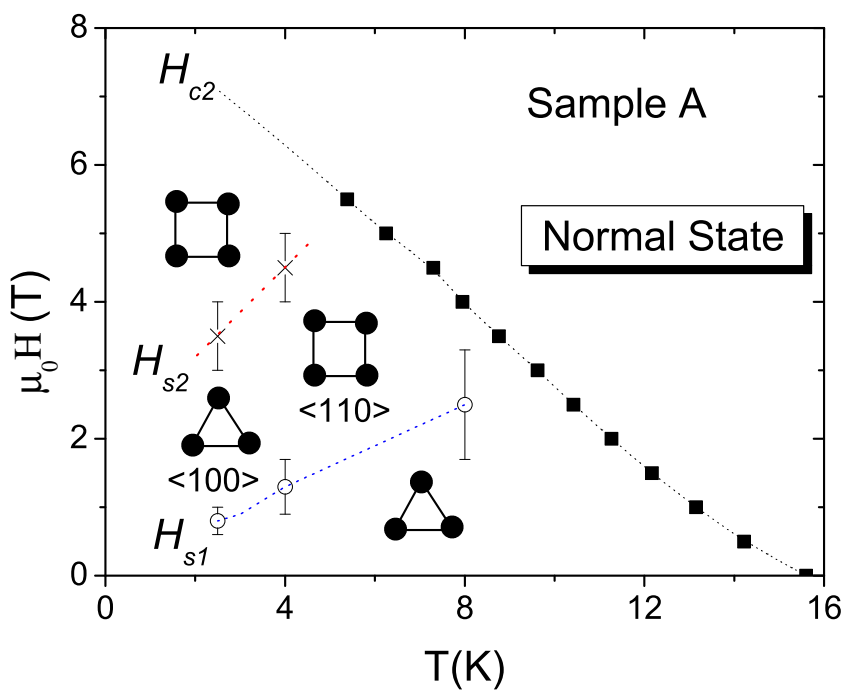

FIG. 11: $H-T$ phase diagram of sample A with lowest $T_{c}$. $H_{s 1}$ is the FLL transition for $H \|[110]$ and $H_{s 2}$ for $H \|[100]$. The dotted lines are guides to the eyes. Triangles and squares were sketched to show the corresponding FLL. ${ }^{38}$

anisotropy $(\Gamma)$ at $2 \mathrm{~K}$ are compared as a function of magnetic field at the bottom panel of Fig. 10. The FLL effect $\gamma$ increases sharply above $0.6 \mathrm{~T}$ and decreases gradually to zero at $4 \mathrm{~T}$, indicating that the low field corresponds to $H_{s 1}$ and the high field to $H_{s 2}$. It is interesting to note that Miranovic et al. came to a similar conclusion by solving quasi-classical Eilenberger equation for nodal superconductors with anisotropic Fermi velocity 45

The $H-T$ phase diagram of the disordered sample A is shown in Fig. 11. $H_{s 1}$ is the FLL transition field for $H \|[110]$ and $H_{s 2}$, for $H \|$ [100]. The increase in the transition fields with increasing temperature is consistent with the nonlocal prediction where the nonlocal range decreases with raising $T: 35$ The difference in the FLL transition fields for $H \|[100]$ and $H \|[110]$ can be due to the upper critical field $H_{c 2}$ anisotropy between the two directions. Since the effective nonlocal range is proportional to the coherence length $\xi$, the $H_{c 2}$ anisotropy leads to a higher FLL transition field for $H \|$ [100] than that for $H \|$ [110]. The FLL transition occurs when the intervortex distance is comparable to the effective nonlocal radius. The observed ratio $H_{s 2} / H_{s 1} \approx 4$ is larger than the predicted ratio $2, \stackrel{35}{=}$ but is smaller than the reported ratio of 10 in $\mathrm{YNi}_{2} \mathrm{~B}_{2} \mathrm{C}{ }^{33}$ The difference between experiments and theory may attest to the need that we take into account both the nonlocality and the anisotropic gap nature of the borocarbides.

\section{CONCLUDING REMARKS}

We reviewed the first direct evidence for a variation of the DOS of nodal quasiparticles (nqp's) of unconventional superconductors $\mathrm{Lu}(\mathrm{Y}) \mathrm{Ni}_{2} \mathrm{~B}_{2} \mathrm{C}$. The four-fold field-angle oscillation in the heat capacity of the borocarbides is due to the nqp's experiencing a field-induced Doppler energy shift. The 3D superconductivity of the borocarbides is reflected in the small oscillation amplitude of $4 \%$, not $40 \%$ expected for 2D superconductors. A dramatic change has also been observed in a slightly disordered Lu1221. A fourfold pattern in $C(\alpha)$ changed to an eightfold or two sets of fourfold pattern at the FLL transition field, evidencing that both nonlocal effects and an anisotropic superconducting gap coexist in the borocarbides.

Even though it is clear that there are nodes along $<100>$ directions in the borocarbides, the type of nodes has yet to be answered. Based on a theoretical calculation, 46 it is asserted that the rapid decrease of the oscillation amplitude of the angle-dependent thermal conductivity with polar angle $\theta$ is due to a point node gap located along [100] ${ }^{15}$ Miranovic et al., however, claimed that the oscillation amplitude decreases sharply with $\theta$ for both vertical line node and the $s+g$ point node cases, suggesting that the conical field rotation experiments may not be able to distinguish the two cases ${ }^{45}$ In order to determine the precise gap structure, instead, they suggested the field dependence of the oscillation amplitudes at low fields. The oscillation monotonically decreases with increasing field for point nodes, while it has a maximum for line nodes. We plan to measure conical field-angle heat capacity and extend our measurements to lower fields, which could shed more light on the controversy of the nature of nodes. A systematic study on disorder effects through Co doping on $\mathrm{Ni}$ site, $\mathrm{LuNi}_{2-x} \mathrm{Co}_{x} \mathrm{~B}_{2} \mathrm{C}$, will clearly elucidate the correlation between nonlocal effects and anisotropic gap effects.

It is gratifying that other field-angle heat capacity have been followed after our pathbreaking work ${ }^{30}$ Deguchi et al. applied the technique to the spin-triplet superconductor $\mathrm{Sr}_{2} \mathrm{RuO}_{4}$ and found a fourfold oscillation for $H \perp c$-axis, providing decisive information on the multiband superconductivity and gap structures ${ }^{47}$ Aoki et al. have studied the heavy-fermion superconductor $\mathrm{CeCoIn}_{5}$ and found a clear fourfold oscillation in $C(\alpha)$ with minima along $\langle 100\rangle$, suggesting the presence of line nodes at those directions $\underline{\underline{48}}$ Their interpretation of the superconducting gap symmetry as $d_{x y}$-wave, however, contradicts that $\left(d_{x^{2}-y^{2}}\right)$ by thermal conductivity data. ${ }^{49}$ It is interesting to note that the angle-resolved specific heat directly measures the zero-energy density of states (ZEDOS). On the other hand, the thermal conductivity data $\kappa(\alpha)$ necessarily involve both the ZEDOS and the quasiparticle scattering time $\tau(\alpha), 11$ making it difficult to identify the gap node direction in some cases.

Finally, we mention that the angle-resolved specific heat measurement opened a new venue to better understanding unconventional superconductivity. Even though only a few classes of unconventional superconductors have been studied by this technique, 30.38 .47 .48 new features on their superconducting properties have been revealed, indicating a whole new aspect of superconductiv- 
ity may await to be found.

\section{Acknowledgments}

Work at Los Alamos was performed under the auspices of the U.S. Department of Energy. Work at University of Illinois was supported by NSF Grant No. DMR 9972087. X-ray measurements were carried out in the Center for Microanalysis of Materials, University of Illinois, which is partially supported by the U.S Department of Energy under grant DEFG02-91-ER45439. TP acknowledges benefits from discussion with Joe D. Thompson.
1 J. Annett, N. Goldenfeld, and A. J. Leggett, Physical Properties of High Temperature Superconductors vol. 5, ed. D. M. Ginsberg (World Scientific, Singapore, 1996).

2 M. Sigrist and K. Ueda, Rev. Mod. Phys 63 (1991) 239.

3 S. K. Yip and J. A. Sauls, Phys. Rev. Lett. 69 (1992) 2264.

4 A. Maeda, Y. Lino, N. Motohira, K.Kishio, and T. Fukase, Phys. Rev. Lett. 74 (1995) 1202.

5 A. Maeda et al., J. Phys. Soc. Jpn. 65 (1996) 3638.

6 A. Bhattacharya et al., Phys. Rev. Lett. 82 (1999) 3132.

7 A. Carrington, R. W. Giannetta, J. T. Kim, and J. Giapintzakis, Phys. Rev. B59 (1999) R14173.

8 C. P. Bidinosti, W. N. Hardy, D. A. Bonn, and R. Liang, Phys. Rev. Lett. 83 (1999) 3277.

9 M. R. Li, P. J. Hirschfeld, and P. Wolfle, Phys. Rev. Lett. 81 (1998) 5640.

10 C. Kubert and P. J. Hirschfeld, Phys. Rev. Lett. 80 (1998) 4693.

11 M. B. Salamon, F. Yu, and V. N. Kopylov, J. Supercon. 8 (1995) 449.

12 F. Yu, M. B. Salamon, and A. J. Leggett, Phys. Rev. Lett. 74 (1995) 5136.

13 H. Aubin, K. Behnia, and M. Ribault, Phys. Rev. Lett. 78 (1997) 2624.

14 K. Izawa et al., Phys. Rev. Lett. 86 (2001) 1327.

15 K. Izawa et al., Phys. Rev. Lett. 89 (2002) 137006.

16 I. Vekhter, P. J. Hirschfeld, J. P. Carbotte, and E. J. Nicol, Phys. Rev. B59 (1999) R9023.

17 K. A. Moler et al., Phys. Rev. Lett. 73 (1994) 2744.

18 Y. Wang, B. Revaz, A. Erb, and A. Junod, Phys. Rev. B63 (2001) 094508.

19 N. D. Whelan and J. P. Carbotte, Phys. Rev. B62 (2000) 14511.

${ }^{20}$ H. Won and K. Maki, Europhys. Lett. 56 (2001) 729.

21 Y. Kraftmakher, Phys. Repts. 356 (2002) 1.

22 P. Sullivan and G. Seidel, Phys. Rev. 173 (1968) 679.

23 T. Park, Ph.D. thesis, University of Illinois, Urbana-
Champaign (2003).

24 R. J. Cava et al., Nature (London) 367 (1994) 252.

25 C. Mazumdar et al., Solid State Commun. 87 (1993) 413.

26 P. C. Canfield, P. L. Gammel, and D. J. Bishop, Phys. Today 51 (1998) 40.

27 M. Nohara, M. Isshiki, H. Takagi, and R. J. Cava, J. Phys. Soc. Jpn. 66 (1997) 1888.

28 G. E. Volovik, JETP Lett. 58 (1993) 469.

29 E. Boaknin et al., Phys. Rev. Lett. 87 (2001) 237001.

30 T. Park, M. B. Salamon, E. M. Choi, H. J. Kim, and S.-I. Lee, Phys. Rev. Lett. 90 (2003) 177001.

31 U. Yaron et al., Nature (London) 382 (1996) 236.

32 M. R. Eskildsen et al., Nature (London) 393 (1998) 242.

33 H. Sakata et al., Phys. Rev. Lett. 84 (2000) 1583.

34 M. R. Eskildsen et al., Phys. Rev. Lett. 86 (2001) 5148.

35 V. G. Kogan et al., Phys. Rev. B55 (1997) R8693.

36 R. Gilardi et al., Phys. Rev. Lett. 88 (2002) 217003.

37 K. O. Cheon et al., Phys. Rev. B58 (1998) 6463.

38 T. Park et al., Phys. Rev. Lett. 92 (2004) 237002.

39 M. Hedo et al., J. Phys. Soc. Jpn. 67 (1998) 272.

40 M. Ichioka, A. Hasegawa, and K. Machida, Phys. Rev. B59 (1999) 184.

41 P. Miranovic, N.Nakai, M. Ichioka, and K. Machida, Phys. Rev. 68 (2003) 052501.

42 K. Maki et al., Europhys. Lett. 64 (2003) 496.

43 P. L. Gammel et al., Phys. Rev. Lett. 82 (1999) 4082.

44 M. R. Eskildsen et al., Phys. Rev. Lett. 86 (2001) 320.

45 P. Miranovic, M. Ichioka, K. Machida, and N. Nakai, cond-mat/0409371, unpublished

46 K. Maki, P. Thalmeier, and H. Won, Phys. Rev. $\mathbf{B 6 5}$ (2002) 140502.

47 K. Deguchi, Z. Mao, H. Yaguchi, and Y. Maeno, Phys. Rev. Lett. 92 (2004) 047002.

${ }^{48}$ H. Aoki et al., J. Phys.: Condens. Matter 16 (2004) L13.

49 K. Izawa et al., Phys. Rev. Lett. 87 (2001) 157002. 